\title{
Performance of ParaHit and OptiMAL tests in the diagnosis of malaria in Mwanza, north-western Tanzania
}

\author{
SIMON N. BUHALATA ${ }^{1 *}$ and JULIUS J. MASSAGA ${ }^{2}$ \\ ${ }^{1}$ National Institute for Medical Research, Mwanza Medical Research Centre, P.O. Box 1462, Mwanza, \\ Tanzania \\ ${ }^{2}$ National Institute for Medical Research, P.O. Box 9653, Dar es Salaam, Tanzania
}

\begin{abstract}
Malaria rapid diagnostic tests (RDTs) are non microscopic tests that provide a rapid detection of malaria infections in infected individuals. The objective of this study was to evaluate the performance of ParaHit and OptiMAL tests for detection of malaria infections as compared with routine microscopy. This facility-based study was carried out in Mwanza, north-western Tanzania and involved outpatients attending Igoma Health Centre. Blood samples were tested for malaria infection using the two RDTs and compared with Giemsa stained blood films examined using microscope. A total of 243 individuals (median age $=22$ years) were involved in the study. Microscopy had a higher detection rate of $19.7 \%$ (48/243) as compared to ParaHit (4.5\%) and OptiMAL (3.7\%). Low sensitivity of $21.2 \%$ and $17 \%$, but high specificity of $99.4 \%$ for ParaHit and OptiMAL, respectively was observed. Of all positive blood slides for Plasmodium falciparum, 78.7\% had low parasite density (80 -720 parasite/ $\mu$ l of blood). These slides were negative for malaria parasite for both RDTs. Over $80 \%$ of study participants who reported fever had negative blood slides for malaria parasites by microscopy. On the other hand, $44.7 \%$ of those who reported no fever had positive blood slides for $P$. falciparum. Study participants who reported to have fever and high parasite density above 720 parasite/ $\mu \mathrm{l}$ were likely to be positive by both RDTs (OR=6.8; $P=$ 0.031529). In conclusion, the overall performance of both RDTs in detecting asexual $P$. falciparum was low as compared to microscopy and their performance were highly affected by parasite density. This calls for further evaluation studies before RDTs are widely used in peripheral health facilities in order to minimize potential severe consequences.
\end{abstract}

Key words: malaria, rapid diagnostic test, sensitivity, specificity, microscopy, Tanzania

\section{Introduction}

Malaria is the major cause of febrile illnesses in tropical countries. It poses a plethora of symptoms that overlap with other febrile illnesses. This overlap of symptoms between malaria and non malarial febrile-illnesses results in significant over-diagnosis of malaria and making decision to treatment difficult (O'Dempsey et al., 1993; Greenwood et al., 1997).

Laboratory methods for diagnosis of malaria pose great challenges for most health facilities in developing tropical countries. For many years, diagnosis of malaria has been done by using microscopy, and this technique has remained the gold standard. Microscopic diagnosis involves taking a blood sample, preparing a stained smear, and examining it for parasites (WHO, 2000). The advantage of microscopy is that it can achieve high sensitivity at low cost if done correctly under quality-controlled conditions with high diagnostic throughput. However, despite of being labour intensive and technical expertise demanding, it also requires quality equipment and reagents and the time lag between blood collection and patient consultation often prevents results from being taken into account in the diagnosis (WHO, 2003; Baker et al., 2005). Sensitivity in malaria diagnosis

\footnotetext{
Simon N Buhalata: E- mail: buhalata@yahoo.com
} 
by microscopy is often low due to the lack of high quality equipment, the use of low quality stains and other reagents, and lack of supervision and trained staff (Mundy et al., 2000; Ishengoma et al., 2010). For example, in East Africa re-evaluations of Giemsa-stained slides revealed that standard reading had a sensitivity of $50 \%-75 \%$ and specificity of $59 \%$ 96\% (Reyburn et al., 2004, 2006; Zurovac et al., 2006).

The recent introduction of non-microscopic malaria tests (rapid diagnostic tests) to detect individuals infected with malaria has opened a new and important step in an effort to correctly diagnose malaria. Rapid diagnostic tests were developed as an alternative to microscopy for malaria diagnosis. These tests detect specific antigens (proteins) produced by asexual malaria parasites that are present in the blood of infected or recently infected individuals (Kakkilaya, 2003). Several commercially available tests are sensitive, specific and, stable under operational conditions (Mboera et al., 2006; Reyburn et al., 2007). These have been developed in recent years, and some are approved by WHO to be used in clinical and research facilities (Kakkilaya, 2003).

Improving diagnosis of acute febrile illnesses so that antimalarial drugs are targeted to patients who need them and alternative diagnosis sought in others is therefore a public health priority especially in Sub-Saharan Africa (Bates et al., 2004). Rapid diagnostic tests have advantages over presumptive treatments in their diagnostic precision and potential to help reduce drug costs due to over-prescription. Rapid diagnostic tests have also advantages over microscopy for use in poorly-resourced areas, particularly when diagnostic throughput is low (Goodman et al., 1999, 2003). The Ministry of Health and Social Welfare in Tanzania is in the process of introducing rapid diagnostic tests for malaria in its primary health care facilities, where most often microscopic diagnosis is unavailable. This study was therefore, carried out to evaluate the performance of ParaHit and OptiMAL tests for detection of malaria infections as compared with routine microscopy.

\section{Materials and Methods}

\section{Study design and population}

This was a cross-sectional study conducted at Igoma Health Centre in Mwanza City in north-western Tanzania. Mwanza City lies on the shores of Lake Victoria at $1200-1500 \mathrm{~m}$ above sea level. Igoma Health Centre has an estimated catchment population of 35,000 people. The study was carried out between November and December 2008. In this study, all attending patients presenting with febrile illnesses were eligible after consenting. Blood samples were collected for preparation of blood films for examination of malaria parasite and RDTs from a finger prick.

The two types of rapid tests used were OptiMAL ${ }^{\circledR}$ and ParaHit ${ }^{\circledast}$. OptiMAL ${ }^{\circledR}$ test (DiaMed AG 1785 Cressiers, Morat, Switzerland) utilizes the enzyme $p$ LDH which is produced by all four species of Plasmodium when present in the blood of infected individuals. ParaHit ${ }^{\circledR}$ test (Span Diagnostics Ltd, India) utilizes HRP-II produced by malaria parasites and released from the infected red blood cells. Malaria rapid tests were performed according to the respective manufacturer's instructions, and were parallel performed on site. Briefly blood from finger prick was drawn into capillary tube and dropped to caste. Buffer solution was added and observed for 15 minutes before reading results. Results were recorded onto a record form. 
Thick and thin blood films were prepared and stained with Giemsa stain for microscopy. Microscopy was done by qualified and competent technicians and $10 \%$ of all blood slides were read by a second reader for quality control at the National Institute for Medical Research, Mwanza laboratory. Parasitaemia density was determined from thick blood smears by counting the number of parasites per 200 white blood cells. The number of parasites counted in the smear was multiplied by 40 to obtain the parasite density per microlitre $(\mu \mathrm{l})$ of blood (Dowling \& Shute, 1966). A slide was declared negative after the examination of 100 high power fields. Thin blood films were used for identification of Plasmodium species.

\section{Data analysis}

Data were entered in MS Excel and analysis was done by using STATA version 10. Proportions, $\mathrm{X}^{2}$ - test and geometric means of parasite densities were calculated. Parasite counts were log-transformed $[\log (x-1)]$ and parasite density reported as geometric mean density (GMD). Statistical significance was reported at $P<0.05$. Sensitivity was calculated as TP/TP+ FN), Specificity TN/TN+FP, Positive Predictive Values (PPV) TP/TP+FP, Negative Predictive Values (NPV) TN/TN+FN, and Accuracy of the test TP+TN/N. Where: N= Total number of study participants; $\mathrm{TP}=$ Proportion of individuals tested positive by both microscopy and RDTs; TN= Proportion of individuals tested negative by both microscopy and RDTs; FP= Proportion of individuals tested false positive by RDTs; FN = Proportion of individuals tested false negative by RDTs.

\section{Ethical consideration}

Ethical clearance was obtained from the Research Committee of The Open University of Tanzania. The permission to conduct the study was obtained from the Mwanza Regional Medical Officer and City Medical Officer of Health. Written consent was obtained from patients and guardians.

\section{Results}

A total of 243 patients presenting with febrile illnesses participated in the study with median age of 22 years (range $=0$ - 75years). The overall proportion of reported fever was $60.9 \%$. Of all subjects, females accounted for majority $(75.7 \%)$ of whom $66.2 \%(98 / 148)$ reported fever. The overall prevalence of malaria as detected by microscopy was $19.7 \%$ (48/ 243) (Table 1). Plasmodium falciparum accounted for the majority (97.3\%) of malaria parasites. Only one slide had $P$. ovale $(1 / 48 ; 2.7 \%)$ and the patient was negative for both OptiMAL and ParaHit tests.

Table1: ParaHit and OptiMAL tests compared with microscopy in detection of malaria

\begin{tabular}{lllll}
\hline Type of RDT & & Microscopy & & \\
& & Positive & Negative & Total \\
\hline ParaHit & Positive & 10 & 1 & 11 \\
& Negative & 37 & 195 & $234(4.7 \%$ \\
OptiMAL & Positive & 8 & 1 & 9 \\
& Negative & 39 & 195 & $234(3.7 \%)$ \\
Total & & 47 & 196 & 243 \\
\hline
\end{tabular}


The prevalence of malaria by ParaHit and OptiMal was 4.5\% and 3.7\%, respectively. The geometric mean parasite density ranged from 80-68500 (mean=2294.3/ $\mu \mathrm{l}$ ) parasites/ $\mu \mathrm{l}$ of blood. Parasitaemia densities were stratified into the strata of 40- 720 (as low); 760-840 (moderate); and $>1000 / \mu$ l (high). Detection thresholds of malaria infections at parasitaemia density were $\geq 760$ and $\geq 840 / \mu l$ (GMPD $\geq 246$ and $\geq 259.2$ ) for ParaHit and OptiMAL tests, respectively. For microscopy, $78.7 \%$ of all positive blood slides had parasite density of $80-$ $720 / \mu \mathrm{l}$ of blood (Table 2). Of individuals reported to have fever, $>80 \%$ had negative blood slides for malaria parasites. However, $44.7 \%$ of those who reported no fever had positive blood slides for $P$. falciparum. Those who had fever and high parasitaemia were likely to be tested positive $(\mathrm{OR}=6.8), P=0.0315$ by both rapid malaria tests. However, in contrary fever and high parasitaemia were not associated with blood slides being positive by microscopy $(P=0.2386)$.

Table 2: The ability of both RDTs to detect asexual malaria parasites stratified at different levels of parasite density

\begin{tabular}{llllll}
\hline RDT & & \multicolumn{4}{c}{ Parasite density level } \\
& & $\mathbf{4 0 - 7 2 0}$ (low) & $\mathbf{7 6 0 - 8 4 0}$ (moderate) & $>\mathbf{> 1 0 0 0}$ (high) & Total \\
\hline ParaHit & Positive & 0 & 2 & 8 & $10(21.2 \%)$ \\
& Negative & 37 & - & - & 37 \\
& Total & 37 & 2 & 8 & 47 \\
\multirow{3}{*}{ OptiMAL } & Positive & 0 & 0 & $8(80 \%)$ & $8(17 \%)$ \\
& Negative & 37 & 2 & - & 39 \\
& Total & 37 & 2 & 8 & 47 \\
\hline
\end{tabular}

The respective sensitivity was $21.2 \%$ and $17.0 \%$. Both tests had a high specificity of $99.4 \%$ (Table 3). The accuracy of test for ParaHit and OptiMAL was 0.84 and 0.83 respectively. Of the age groups, P. falciparum malaria was detected (33.3\%) highest among young children (<10years) and, 2.1\% lowest among the elderly (>60years) by microscopy (Figure1).

Table 3: Sensitivity, specificity, positive and, negative predictive values of ParaHit and OptiMAL tests for malaria detection. (CI $95 \%$ )

\begin{tabular}{lllll}
\hline RDT & Sensitivity (CI) & Specificity (CI) & PPV (CI) & NPV (CI) \\
\hline \multirow{3}{*}{ ParaHit } & $21.2 \%(10 / 47)$ & $99.4 \%(10 / 11)$ & $90.9 \%(10 / 11)$ & $84.4 \%(196 / 232)$ \\
& $($ CI $7.9,27.3)$ & $($ CI $96.9,100)$ & $($ CI $58.7,99.8)$ & $($ CI $71.2,82.4)$ \\
OptiMAL & $17 \%(8 / 47)$ & $99.4 \%(8 / 9)$ & $88.9 \%(8 / 9)$ & $83.7 \%(196 / 234)$ \\
\hline
\end{tabular}
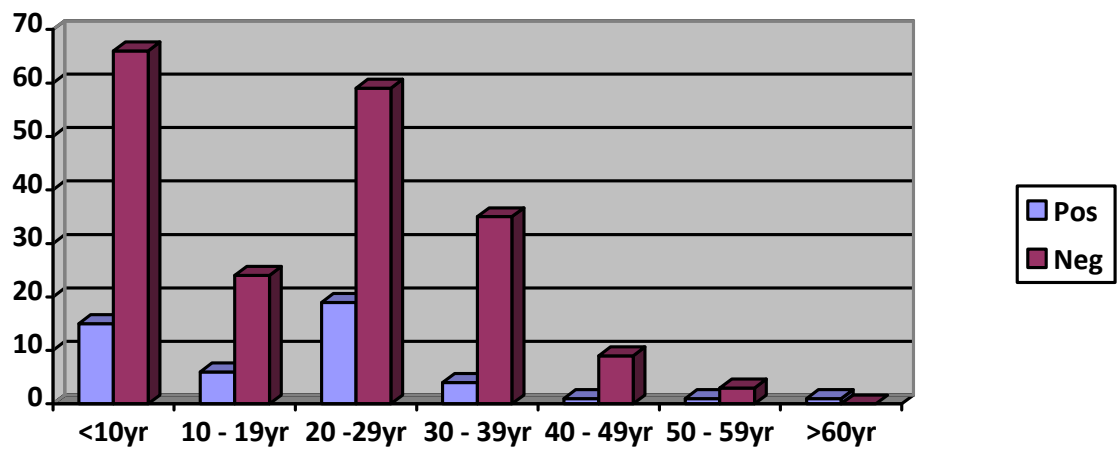

Figure 3: Distribution of malaria infection among age groups as detected by microscopy 


\section{Discussion}

The overall prevalence of asexual $P$. falciparum malaria detected by both RDTs was found to be low as compared to microscopy. Both the two rapid tests showed a very low sensitivity but very high specificity in our study. Similar low prevalence of asexual $P$. falciparum malaria by microscopy and ParaHit test and low sensitivity were observed in another study in the same region in Tanzania (Kamugisha et al., 2009) and in Philippines (Belizario et al., 2005). In contrary, studies using ParaHit-f test in India showed higher sensitivity (Neeru et al., 2005). Using Paracheck-Pf in other areas of Tanzania sensitivities and specificities of $>90 \%$ have been reported (Mboera et al., 2006; Kamugisha et al., 2008).

Low parasite levels are more frequently found in low transmission areas. In our study, microscopy detected asexual parasites in patients with parasitaemia density ranged from 40- 68500 parasites/ $\mu \mathrm{l}$, but a high proportion of those with positive blood slides had low asexual parasite densities below detection thresholds of RDTs. Both RDTs could not detect low parasite count of $<760$ parasites/ $\mu$ l. Low sensitivity of malaria rapid test (PfHRP2) has also been reported elsewhere (Neeru et al., 2005; Mboera et al., 2006).

Fever and high parasite density were found to have a strong association with positive RDTs in this study. However, microscopy detected high asexual $P$. falciparum malaria infections among individuals who reported no fever. This indicates that asymptomatic infections are high in this area, because during low malaria transmission some individuals might be expected to have a high asymptomatic parasitaemia, a low threshold of parasite density which has been not suggested to reflect clinical malaria better. The sensitivity of the pLDH test in field studies has also been reported to be lower at low parasitaemia in field studies (Baker et al., 2005). Generally, RDTs achieve a sensitivity of $>90 \%$ at high parasite densities $>1000$ parasites/ $\mu$ l and, the sensitivity decreases markedly below that level of parasite density (Coleman et al., 2004; Goodman et al., 2006). Further, RDTs have been reported to give false negative results even at higher levels of parasitaemia. Therefore, in cases of suspected severe malaria or complex health emergencies, a positive result may be confirmatory but a negative result may not rule out malaria, and should always be confirmed by microscopy (Reyburn et al., 2007).

This study has a number of limitations. This was a cross-sectional study, generalizability of results could not been possible because of study population was at high risk of other non malarial febrile- illnesses, which could affect generalizability of results. Positive results with RDTs do not rule out other possible causes of febrile illnesses such as relapsing fever. False positive results may be due to gametocytemia, persistent viable asexual-stage parasitaemia below the detection limit of microscopy possibly due to drug resistance, persistence of antigens due to sequestration and incomplete treatment, delayed clearance of circulating antigens and cross reaction rheumatoid factor. False negative tests have been observed even in severe malaria with parasitaemia $>40000$ parasites $/ \mu$. This has been attributed to possible genetic heterogeneity of PfHRP2 expression, deletion of HRP-2 gene, presence of blocking antibodies for PfHRP2 antigen or immune-complex formation, prozone phenomenon at high antigenemia or to unknown causes.

In conclusion, the overall performance of both RDTs in detecting asexual $P$. falciparum was low as compared to microscopy and their performance were highly affected 
by parasite density calling for further evaluation studies before its wide use in peripheral health facilities in order to minimize potential severe consequences.

\section{Acknowledgements}

We are grateful to all who participated in this study at Igoma Health Centre. We would like also to extend thanks to the laboratory staff at the National Institute for Medical Research in Mwanza for the support in the examination of blood smears. This study was supported by the National Institute for Medical Research, Tanzania.

\section{References}

Baker, J., McCarthy, J. Gatton, M., Kyle, D.E., Belizario, V., Luchavez, J., Bell, D. \& Chang, Q. (2005) Genetic diversity of Plasmodium falciparum histidine-rich protein 2 (PfHRP2) and its, effect on the performance of PfHRP2 - based rapid diagnostic test. Journal of Infection and Disease 192,870-877.

Bates, I., Bekoe, V. \& Asamoa-Adu, A. (2004) Improving the accuracy of malaria-related laboratory tests in Ghana. Malaria Journal 3:38.

Belizario, V.Y., Pasay, C.J., Bersabe, M.J., WU de Leon, Guerrero, D.M. \& Bugaoisan, V.M., (2005) Field evaluation of malaria rapid diagnostic tests for the diagnosis of $P$. falciparum and non-P. falciparum infections. Southeast Asian Journal of Tropical Medicine and Public Health 36, 552-561.

Coleman, P.G., Morel, C., Shillcutt, S., Goodman, C. \& Mills, A.J. (2004) A threshold analysis of the cost-effectiveness of artemisinin-based combination therapies in subSaharan Africa. American Journal of Tropical Medicine and Hygiene 71(Suppl 2), 196204.

Dowling, M.A \& Shute G.T (1966) A comparative study of thick and thin blood films in the diagnosis of scanty malaria parasitaemia. Bulletin of the World Health Organization 34, 249-267.

Goodman, C.A., Mutemi, W.M., Baya, E.K., Willetts, A. \& Marsh, V. (2006) The costeffectiveness of improving malaria home management: shopkeeper training in rural Kenya. Health Policy Planning21, 275-288.

Goodman, C.A. (1999) The Economic Evaluation of Malaria Diagnosis: Working paper prepared for informal consultation on "Malaria diagnostics at the turn of the century". Geneva, WHO and USAID: 18.

Greenwood, B.M. (1997) The epidemiology of malaria. Annals of Tropical Medicine and Parasitology 91, 763-769.

Ishengoma, D.R.S., Derua, Y.A., Rwegoshora, R.T., Tenu, F., Massaga, J.J., Mboera, L.E.G., G., Magesa, S.M. (2010) The performance of health laboratories and the quality of malaria diagnosis in six districts of Tanzania. Annals of Tropical Medicine and Parasitology 104, 123-135.

Kakkilaya, B.S. (2003) Rapid diagnosis of malaria. Laboratory Medicine 8, 602- 608.

Kamugisha, M.L., Msangeni, H., Beale, E., Malecela, E.K., Akida, J., Ishengoma, D.R.S. \& Lemnge, M.M. (2008) Paracheck-Pf compared with microscopy for diagnosis of Plasmodium falciparum malaria among children in Tanga City, north-eastern Tanzania. Tanzania Journal of Health Research 10, 14-19. 
Kamugisha, E., Mazigo, H., Manyama, M., Rambau, P., Mirambo, M., Kataraihya, J.B. \& Mshana, S. (2009) Low sensitivity but high specificity of ParaHit $\mathrm{f}$ test in diagnosing malaria among children attending outpatient department in Butimba District Hospital Mwanza, Tanzania. Tanzania Journal Health Research 11, 97-99.

Mboera, L.E.G., Fanello, C.I., Malima, R.C., Talbert, A., Fogliati, P., Bobbio, F. \& Molteni, F. (2006) Comparison of the Paracheck-Pf test to microscopy for confirmation of Plasmodium falciparum malaria in Tanzania. Annals of Tropical Medicine E Parasitology 100, 115-122.

Mundy, C., Ngwira, M., Kadewele, G., Bates, I., Squire, S.B. \& Gilks, C.F. (2000) Evaluation of microscope condition in Malawi. Transactions of the Royal Society of Tropical Medicine and Hygiene 94, 583-584.

Neeru, S., Mishara, A.K., Shukla, M.M., Chand, S.K. \& Praveen, K.B. (2005) Diagnostic and prognostic utility of an inexpensive rapid onsite malaria diagnostic test (ParaHIT-f) among ethnic tribal population in areas of high, low and no transmission in central India. BMC Infectious Disease 5, 50.

O'Dempsey, T.J., McArdle, T.F., Laurence, B.E., Lamont, A.C., Todd, J.E. \& Greenwood, B.M. (1993) Overlap in the clinical features of pneumonia and malaria in African children. Transactions of the Royal Society of Tropical Medicine and Hygiene 87, 662-665.

Peters, R. P. H., Zijlstra, E.E., Schijffelen, M.J., Walsh, A.L., Joaki, G., Kumwenda, J.J., Kublin, J.G., Molyneux, M.E. \& Lewis, D.K. (2004) A prospective study of bloodstream infections as cause of fever in Malawi: clinical predictors and implications for management. Tropical Medicine \& International Health 9, 928-934.

Redd, S.C., Bloland, P.B., Kazembe, P.N., Patrick, E., Tembenu, R. \& Campbell, C.C. (1992) Usefulness of clinical case-definitions in guiding therapy for African children with malaria or pneumonia. Lancet 340, 304-305.

Reyburn, H., Mbatia, R., Drakeley, C., Carneiro, I., Mwakasungula, E., Mwerinde, O., Saganda, K., Shao, J., Kitua, A., Olomi, R., Greenwood, B.M. \& Whitty, C.J. (2004) Overdiagnosis of malaria in patients with severe febrile illness in Tanzania: a prospective study. BMJ 329, 1212.

Reyburn, H., Mbakilwa, H., Mwangi, R., Mwerinde, O., Olomi, R., Drakeley, C. \& Whitty, C. J. (2007) Rapid diagnostic tests compared with malaria microscopy for guiding outpatient treatment of febrile illness in Tanzania: randomized trial. BMJ 334, 403.

Reyburn, H., Ruanda, J., Mwerinde, O. \& Drakeley, C. (2006) The contribution of microscopy to targeting antimalarial treatment in a low transmission area of Tanzania. Malaria Journal 5:4.

WHO (2000) New perspectives malaria diagnosis. World Health Organization, Geneva, WHO/MAL/2000.14: 57.

WHO (2003) Malaria rapid diagnosis, making it work: Informal consultation on field trials and quality assurance on malaria rapid diagnostic tests. Manila, RS/2003/GE/05(PHL): 61.

Zurovac, D., Midia, B., Ochola, S.A., English, M. \& Snow, R.W. (2006) Microscopy and outpatient malaria case management among older children and adults in Kenya. Tropical Medicine \& International Health 11, 432-40. 